\title{
Contribución al conocimiento geológico de la Serranía del Perijá mediante cartografía a escala 1:25.000 del Grupo Cogollo, en el sector oriental del municipio de Becerril, Cesar
}

\author{
Contribution to the geological knowledge of the Serranía del Perijá \\ mountain range through 1:25,000 scale mapping of the Cogollo Group \\ in the eastern sector of the municipality of Becerril, Cesar
}

\begin{abstract}
Milton David Lozada Molina ${ }^{1}$, Frank David Lascarro Navarro ${ }^{1}$, Luisa Fernanda León Díaz ${ }^{1}$, Elías Ernesto Rojas Martínez ${ }^{1}$, Fabio Enrique Carrillo Bolaño ${ }^{1}$, Luis Carlos Tapia Vela ${ }^{1}$, Dino Carmelo Manco Jaraba²

Email de correspondencia: erojas@areandina.edu.co
\end{abstract}

Recibido: 10 de julio de 2018

Aceptado: 12 de marzo de 2019

Publicado en línea: 15 de julio de 2019

Doi: 10.32685/0120-1425/boletingeo.45.2019.487

Citación: Lozada M., M. D. et al. (2019). Contribución al conocimiento geológico de la Serranía del Perijá mediante cartografía a escala 1:25.000 del Grupo Cogollo, en el sector oriental del municipio de Becerril, Cesar. Boletín Geológico, 45, 49-59. Doi: https://doi.org/10.32685/0120-1425/boletingeo.45.2019.487

\section{RESUMEN}

En el departamento del Cesar, municipio de Becerril, se encuentran rocas Cretácicas de composición calcárea que afloran en gran parte de su territorio y pertenecen a la Formación Lagunitas y Aguas Blancas, del Grupo Cogollo, y la Formación La Luna. Este estudio presenta los resultados de un trabajo cartográfico que tiene como objetivo contribuir al conocimiento geológico de esta zona, localizada en un área de $25 \mathrm{~km}^{2}$ en el sector oriental del municipio de Becerril, mediante labores de campo, identificación de unidades geomorfológicas y descripciones petrográficas a escala 1:25.000. Se concluye que la Formación Lagunitas se caracteriza por presentar una sucesión de calizas masivas fosilíferas de coloración gris oscura, con pequeñas alternaciones de lodolitas calcáreas y calizas lumaquélicas. De la Formación Aguas Blancas se identifica con detalle el Miembro Ánimas, constituido por lodolitas calcáreas con abundante materia orgánica, intercalada con calizas micríticas de color negro, que gradan horizontalmente a arenis-

Fundación Universitaria del Área Andina.

Universidad de la Guajira. 
cas con cemento calcáreo de grano fino; el Miembro Tucuy, compuesto por limolitas arenosas de cemento calcáreo, areniscas de gran fino y arcillolitas carbonatadas, y el Miembro Maracas, constituido principalmente por calizas lumaquélicas masivas. De igual forma, se identificaron cuatro ambientes geomorfológicos: estructural, en el que se identificaron las unidades de barras monoclinales, ladera estructural de sierra homoclinal y ladera de contrapendiente de sierra homoclinal; denudacional, en el que se identificaron unidades como escarpe de erosión, lomeríos poco bisectados y ondulaciones denudacionales; cárstico, con unidades de valle cárstico y ladera cárstica; y fluvial, con la unidad llanura de inundación.

Palabras clave: Cartografía, Grupo Cogollo, Serranía del Perijá.

\begin{abstract}
A B S T RACT
In the department of Cesar, Municipality of Becerril, there are extensive outcroppings of Cretaceous rocks with calcareous sequences belonging to the Lagunitas and Aguas Blancas Formations, the Cogollo Group, and the La Luna Formation. This study presents the mapping of a $25 \mathrm{~km}^{2}$ are in the eastern sector of the Municipality of Becerril, intended to contribute geological knowledge of this area through field work, identification of geomorphological units and petrographic descriptions at a scale 1:25,000. The Lagunitas Formation is characterized by a succession of dark gray massive fossiliferous limestone intercalated with calcareous mudstone and lumachellic limestone. From the Aguas Blancas Formation, the Ánimas Member is identified in detail, consisting of calcareous mudstone with abundant organic matter interbedded with black micritic limestones that grades horizontally to sandstones with fine-grained calcareous cement. The Tucuy Member consists of calcareous siltstone, fine-grained sandstone and carbonated claystone. The Maracas Member consists mainly of massive lumachellic limestone. Similarly, four geomorphological environments were identified: structural, identifying units of hog-backs, homoclinal ridges and cuestas; denudation, identifying units such as erosion scarps, lightly bisected hills and undulating interfluves; karstic, with units of karstic valley and karstic slope; and fluvial, with the floodplain unit.
\end{abstract}

Keywords: Cartography, Cogollo Group, Serranía del Perijá.

\section{INTRODUCCIÓN}

a Serranía del Perijá es conocida como un sistema montañoso localizado en la esquina noroccidental de Suramérica, levantada entre finales del Eoceno y el Plioceno por actividad orogénica debido a la colisión de las placas de Suramérica y el Caribe (Cáceres, Cediel, Ujueta, 1976).

Este trabajo se desarrolló sobre el flanco occidental de la Serranía del Perijá, donde afloran rocas cretácicas sedimentarias pertenecientes a la Formación Río Negro, el Grupo Cogollo, y la Formación La Luna. El área cartografiada se localiza al nororiente del municipio de Becerril, en la carretera que comunica el corregimiento de Casacará con la Jagua de Ibirico, sector atravesado por los ríos Maracas y Tucuy, que se intersecan con las quebradas Socomba y Rodrigo (figura 1).

Los resultados de la cartografía a escala 1:25.000 del Grupo Cogollo son una contribución al conocimiento geológico de la Serranía del Perijá en este sector, debido a que los estudios cartográficos y petrográficos a dicha escala son desconocidos o escasos. 


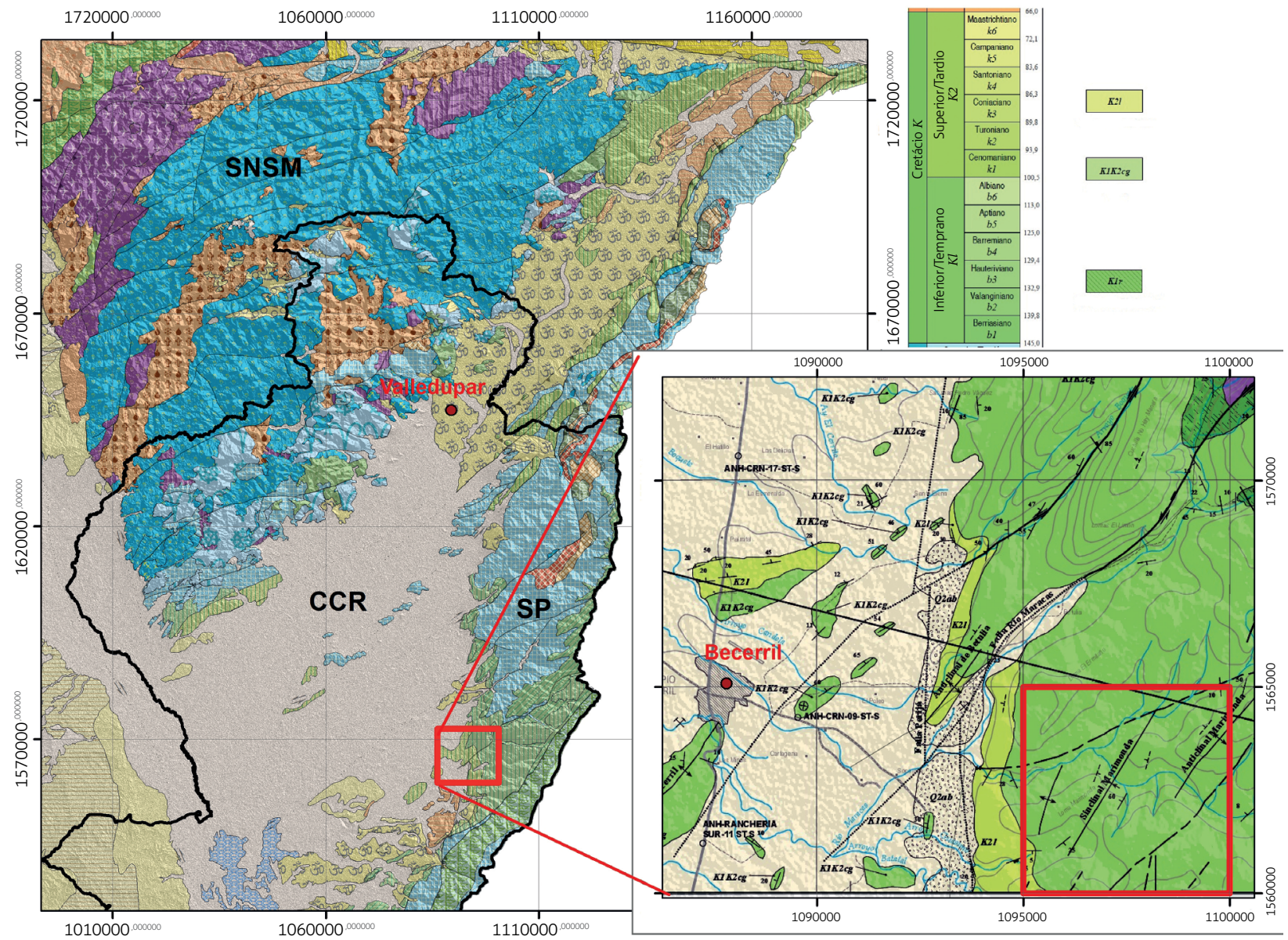

Figura 1. Mapa de localización. SNSM: Sierra Nevada de Santa Marta; CCR: cuenca Cesar-Ranchería; SP: Serranía del Perijá. Fuente: adaptado y modificado a partir de la Plancha 41-Becerril, SGC (2015)

\section{Metodología y procedimientos}

La presente metodología se basa en la "Metodología de la investigación" de Hernández S. (2014) y tiene un enfoque cuantitativo, ya que busca describir, explicar y comprobar los datos obtenidos en campo para adelantar un análisis objetivo, con alcances descriptivos, correlacionales y explicativos que resultan de la revisión bibliográfica, al tiempo que brinda perspectivas para la cartografía. Asimismo, cuenta con un diseño no experimental basado en el análisis de la documentación relacionada con el tema de estudio, el levantamiento y la digitalización de los mapas correspondientes y la sistematización de los datos obtenidos en campo, donde se realizó muestreo de tipo no probabilístico, ya que las muestras fueron obtenidas de forma intencional por los cambios de litologías.

Este estudio comprendió varias etapas o fases en las que se logró un correcto desarrollo del trabajo: primero se reconocieron unidades geológicas de la zona mediante un estudio fotogeológico (fotografías aéreas 200, 201, 202, del vuelo C2506, y fotografías 12, 13, 14, 15, del vuelo C2435, correspondientes a la plancha 41IVC del Instituto Geográfico Agustín Codazzi [IGAC]). Se realizó una visita a campo que se correlacionó con geología regional encontrada en la memoria geológica del Cesar; también se realizaron estudios cartográficos en la región. Posteriormente se realizaron descripciones petrográficas de las muestras recolectadas y levantamiento estratigráfico de las unidades en la zona, y se procedió a identificar con claridad la distribución geográfica de cada miembro del Grupo Cogollo y los depósitos del Cuaternario. Asimismo, se tomaron datos estructurales con brújula en los flancos de los pliegues encontrados, que permitieron la finalización de la cartografía del área. 


\section{Geología regional}

La zona de trabajo se ubica sobre dos provincias geológicas: el piedemonte occidental de la Serranía del Perijá y el oriente de la cuenca Cesar-Ranchería.

El municipio de Becerril se caracteriza por presentar rocas de diferentes ambientes geológicos, con contrastes en litología, edad y composición. A escala 1:100.000 sobre la Plancha 41, González et al. (2015) dan información de la geología regional, en la que se pueden apreciar rocas metamórficas y formaciones sedimentarias (figura 1).

\subsection{Rocas metamórficas}

Las rocas metamórficas están representada en el ámbito regional por la granulita de Los Mangos, que tiene presencia en la parte más alta de la Serranía del Perijá, hacia el este del municipio de Codazzi (Colmenares et al., 2007).

\subsection{Rocas sedimentarias}

En la Serranía del Perijá afloran rocas sedimentarias de gran diversidad, como areniscas, lodolitas, lutitas y calizas, entre otras. Además, estas rocas hacen parte de formaciones de importancia minera en la región, como las Formaciones Barcos y Los Cuervos (esta última contiene mantos de carbón explotables). Aparte de ello, en la zona de estudio afloran las Formaciones Río Negro, Lagunitas, Aguas Blancas y La Luna, así como los depósitos del Cuaternario, con espesores que pueden ser de interés para la explotación minera (Gil y Martínez, 1990; Hernández, 2003).

\subsubsection{Formación Río Negro}

La Formación Río Negro, inicialmente descrita por Liddle (1928), comprende areniscas, conglomerados, lodolitas y arcillolitas, de edad Neocomiano a Aptiano. Las areniscas generalmente se presentan con coloración blanca y grano grueso; los conglomerados, heterogéneos; las arcillolitas y lodolitas típicamente muestran tonos brillantes de amarillo, rojo y morado por presencia de óxidos de hierro, composicionalmente son arcosas a subarcosas y su espesor varía entre 800 y 1.000 metros en la zona cercana a Fernambuco; esta se encuentra en contacto gradacional con el Grupo Cogollo (Geoestudios, 2006; González et al., 2015; González et al., 2008).

\subsubsection{Grupo Cogollo}

El Grupo Cogollo fue descrito inicialmente por Miller (1960), quien agrupó una serie de calizas, calizas arenosas y areniscas calcáreas de edad Barremiano-Aptiano que hacen parte del bloque levantado del borde occidental de la Serranía de Perijá. Su contacto inferior es discordante con la Formación La Quinta y gradacional con la Formación Río Negro (Castro, 2009; González et al., 2008). Litológicamente, este grupo es dividido de base a techo en las Formaciones Lagunitas y Aguas Blancas, y esta última es subdividida en tres miembros por Hernández (2003) y González et al. (2008): Ánimas, Tucuy y Maracas.

\subsubsection{Formación La Luna}

La Formación La Luna es descrita por Gardner (1926), quien la describe como una secuencia alternante de limolitas, arcillolitas, lutitas negras carbonosas, calizas bituminosas, capas de chert y nódulos de variable tamaño. Hacia la base de la formación predominan las lodolitas y las capas de chert, mientras que en la parte superior son comunes las calizas bituminosas. Además, esta formación es concordante con el Grupo Cogollo y transicional con la Formación Molino. La edad de la Formación La Luna, según informes de la Agencia Nacional de Hidrocarburos (González et al., 2008) se ubica entre el Turoniano Temprano y el Coniaciano.

\subsubsection{Depósitos del Cuaternario}

Están constituidos por acumulaciones de sedimentos no consolidados de origen fluvial.

A escala 1:100.000, González et al. (2008) señalan que en el área de estudio se observan rocas de edad Cretácica, pertenecientes al Grupo Cogollo y a Formación La Luna. Debido a la escala de trabajo que manejaron no les fue posible separar el Grupo Cogollo en sus formaciones y subunidades.

\section{Cartografía}

En el área afloran unidades litológicas con edades que van desde el Aptiano al Coniaciano, según estudios anteriores proporcionados por Geoestudios (2006) y Gonzales et al. (2015). Todas las muestras se tomaron en un recorrido marcado con GPS que se visualiza en la figura 2, en las que se encontraron rocas de las Formaciones Lagunitas y Aguas Blancas, descritas a continuación. 

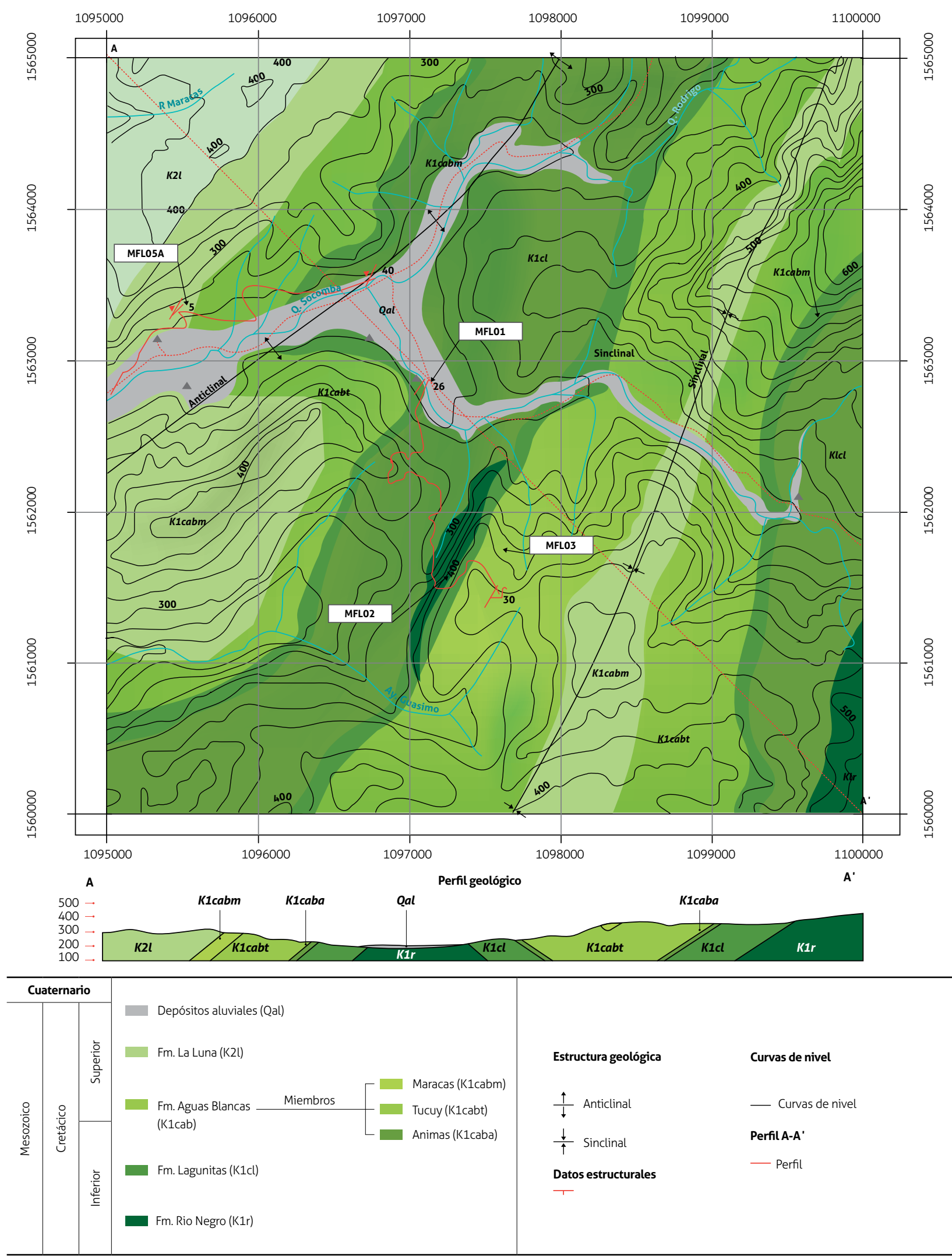

Figura 2. Cartografía geológica levantada, con las localizaciones de las muestras recolectadas en campo. Fuente: autores 


\subsection{Formación Lagunita}

Se caracteriza por presentarse como una sucesión de calizas masivas fosilíferas de coloración gris oscura, con pequeñas alternaciones de lodolitas calcáreas y calizas lumaquélicas (Hernández, 2003), cuyo espesor máximo alcanza los 290 metros (Cáceres et al., 1976).

$\mathrm{El}$ área de estudio se encuentra representada por la muestra MFL01, descrita como una caliza de color negro grisáceo a marrón claro, con presencia de venillas de calcita, de textura masiva, catalogada en la tabla de clasificación de Dunham (1962) como mudstone, y según Folk (1962), como una micrita con intraclastos. La muestra, por sus características litológicas y ubicación geográfica, se asocia a la Formación Lagunita (figura 3).

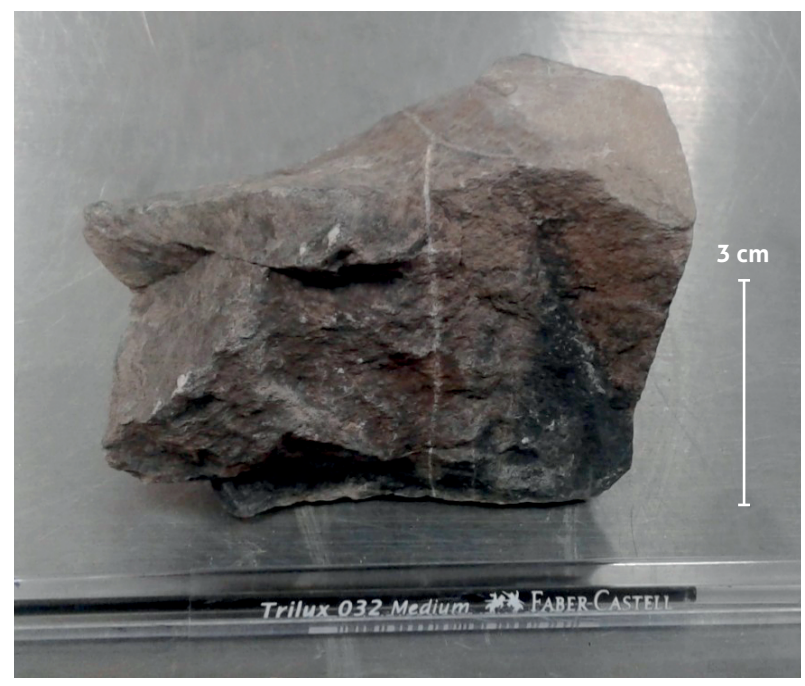

Figura 3. Muestra de caliza perteneciente a la Formación Lagunita Fuente: autores

\subsection{Formación Aguas Blancas}

Está constituida por tres Miembros (Ánimas, Tucuy y Maracas), de los cuales se identificaron y describieron los de Ánimas y Tucuy.

\subsubsection{Miembro Ánimas}

El Miembro Ánimas está constituido por un conjunto lodolítico calcáreo con abundante materia orgánica intercalado con calizas micríticas de color negro que gradan horizontalmente a areniscas con cemento calcáreo de grano fino; su espesor es de 285 metros (González et al., 2008).
En el área de estudio, el Miembro Ánimas se encuentra en afloramientos en los que se observan lutitas bituminosas con intercalaciones de limolitas calcáreas de color gris, con laminación plano-paralelas (figura 4). Estas rocas se asocian a la Formación Aguas Blancas, Miembro Ánimas, por su correlación litológica y características muy similares a las descritas en campo y en el laboratorio. La muestra MFL 02 es descrita microscópicamente, según la clasificación de Dunham (1962), como un wackestone lodo-soportado (bitumen como matriz de la roca "Btm"), y en ella se observan diversas especies de foraminíferos planctónicos de caparazón micrítico, así como foraminíferos fragmentados (globigerinas) "Fr". En dos partes de la sección se observan vetillas de calcita recristalizada que atraviesa de forma horizontal la sección (figura 5).

Según la clasificación de Flügel (2004) sobre la distribución de los tipos de microfacies, presenta microfacies SMF-3 (wackestone con foraminíferos), lo que permite relacionar el Miembro Animas a un ambiente de depositación de pie de talud, en rampa carbonatada exterior (FZ-3), según Wilson (1975).

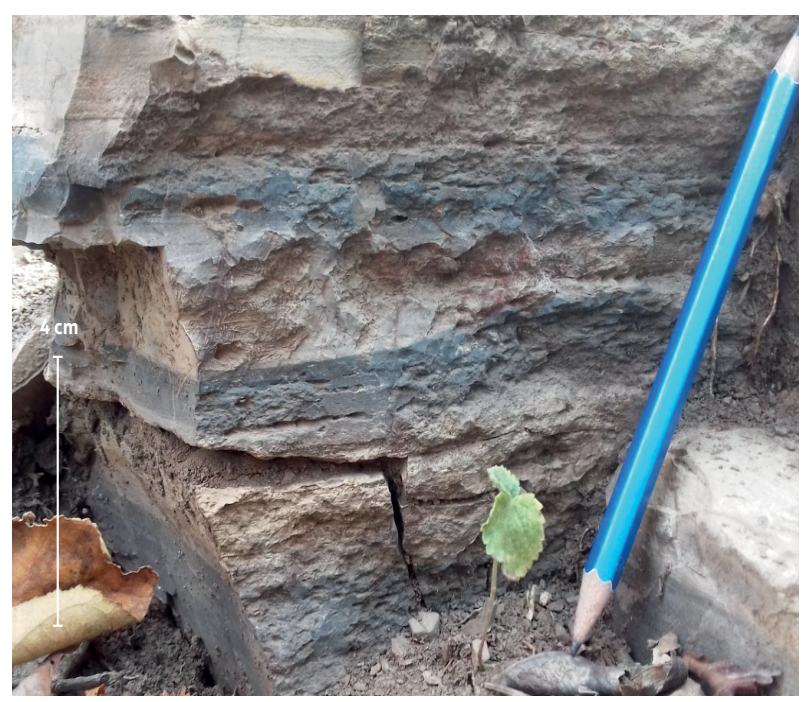

Figura 4. Formación Aguas Blancas, Miembro Ánimas. Intercalaciones de lutitas oscuras con limolitas calcáreas.

Fuente: autores 

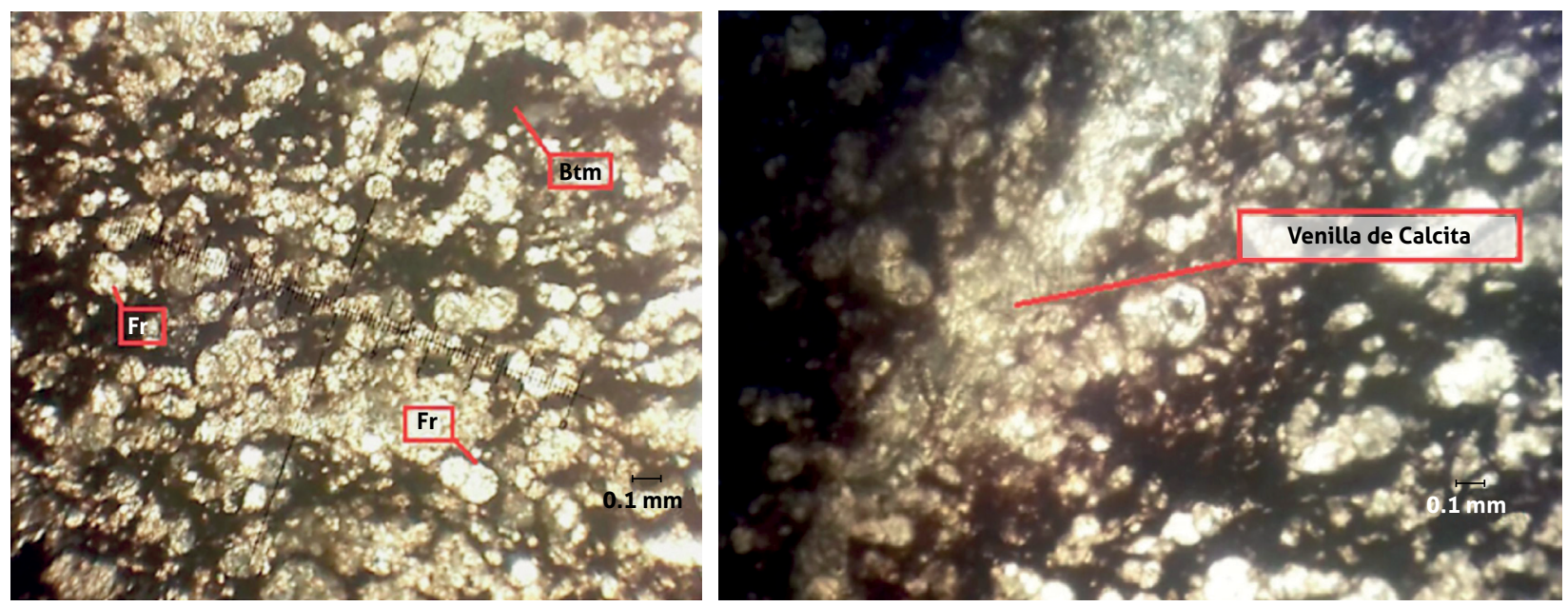

Figura 5. Fotomicrografía de lámina delgada de wackestones pertenecientes a la Formación Aguas Blancas, Miembro Ánimas. Luz reflejada; ampliación: 10x.

Fuente: autores

\subsubsection{Miembro Tucuy}

El Miembro Tucuy agrupa un conjunto arenoso presente en la Formación Aguas Blancas, constituido por limolitas arenosas de cemento calcáreo, areniscas de grano fino y arcillolitas calcáreas. Su espesor es de 450 metros, según González et al. (2008).

La muestra MFL03 se clasifica como una arenisca lodosa (>90\% arena) de color amarillo ocre, en algunas partes gris de grano fino a muy fino si se observa con ayuda del estereomicroscopio (es difícilmente perceptible a simple vista) y con patrones texturales proporcionados (Castro, 1989). Se observa matriz arenosa con cemento carbonatado, con buen calibrado, granos subangulares a subredondeados, con empaquetamiento completo y sorting de 0,35. Presenta una estratificación plano-paralela. La muestra está compuesta en un 95\% de cuarzo (Qz), $1 \%$ de feldespatos (Fd), 1\% de fragmentos líticos (Fr), y $3 \%$ de minerales accesorios. Según Folk (1974), se clasifica como una cuarzoarenita, y se relaciona con el Miembro Tucuy, cuyo ambiente tectónico procede de bloques continentales de cratón interior, según la clasificación de Dickinson (1985) (figura 6).

La muestra MFL05A, tomada en campo, es una caliza arenosa de color amarillo ocre con oxidación en algunas partes. Tiene una laminación ondulada de textura masiva y es clasificada, según Dunham (1962), como wackestone, y según Folk (1962), como una micrita con fósiles (figura 7).

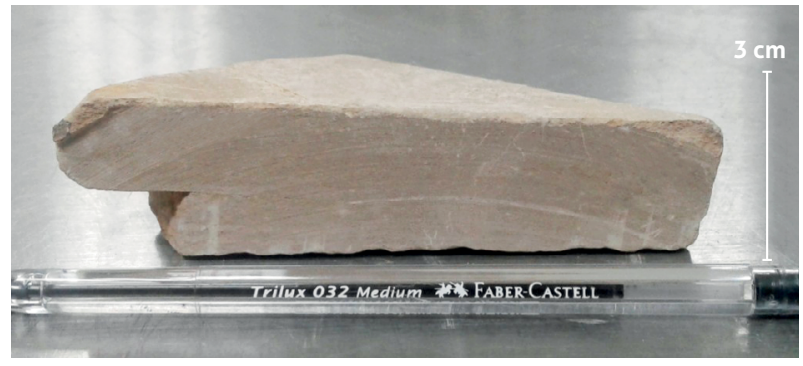

Figura 6. Muestra MFL03 de arenisca perteneciente a la Formación Aguas Blancas, Miembro Tucuy.

Fuente: autores

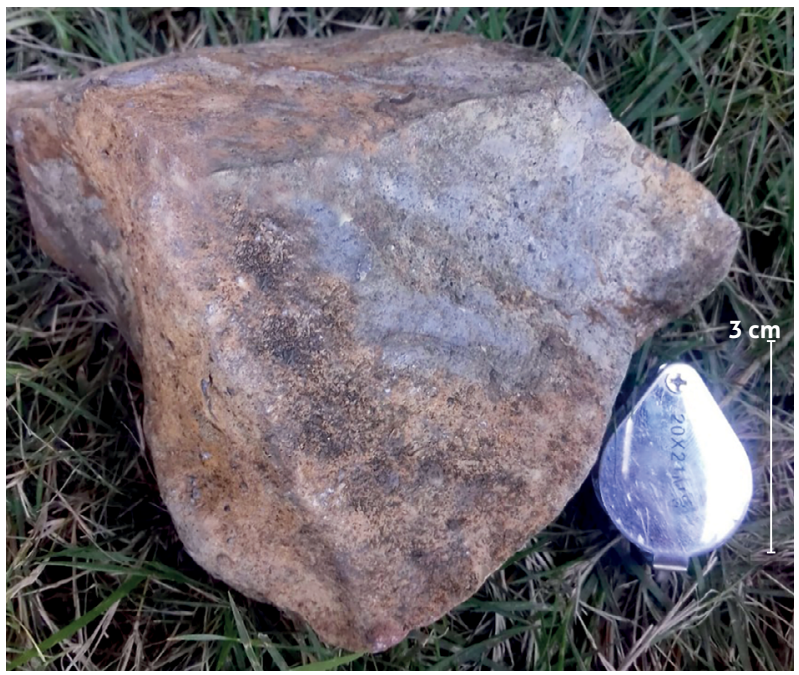

Figura 7. Muestra MFL5A, caliza arenosa perteneciente a la Formación Aguas Blancas, del Miembro Tucuy.

Fuente: autores 


\subsubsection{Miembro Maracas}

El Miembro Maracas es el conjunto calcáreo superior de la Formación Aguas Blancas, y está compuesto principalmente por calizas lumaquélicas masivas. El espesor de este Miembro es de 217 metros (González et al., 2008).

\section{Geomorfología}

La geomorfología se llevó a cabo con información recogida en campo y fotogeología, en las que se identificaron nueve unidades geomorfológicas, que son agrupadas en cuatro ambientes geomorfológicos: estructural, denudacional, cárstico y fluvial (figura 8).

\subsection{Ambiente estructural}

\subsubsection{Barras homoclinales (Sbh)}

Caracterizadas por presentarse como una tendencia alargada. En su morfología se destaca la alternancia en la disposición subvertical de las unidades resistentes, si se compara con las superficies suaves de las zonas excavadas, fenómeno debido a procesos de escorrentía en las unidades blandas.

\subsubsection{Ladera estructural de sierra homoclinal (Sshle)}

Se caracteriza por la inclinación de los estratos a favor de la pendiente. Su longitud es corta, y sus formas son rectas y con pendientes poco escarpadas. Se relaciona con a una estructura homoclinal.

\subsubsection{Ladera de contrapendiente de sierra homoclinal (Sshlc)}

En el área de estudio, este tipo de ladera se caracteriza por presentarse como una superficie subvertical de escasa longitud, de forma cóncava, con pendiente abrupta a escarpada. Es generada por estratos dispuestos contra la pendiente del terreno y se relaciona con una estructura de sierra homoclinal.

\subsection{Ambiente denudacional}

\subsubsection{Escarpe de erosión (Deeme)}

Localizado en un altiplano, el escarpe de erosión es definido como una unidad geomorfológica de forma recta con pendiente escarpada, de ladera abrupta, originada por socavación fluvial lateral o por procesos de erosión y movimientos en masa remontantes a lo largo de un drenaje.

\subsubsection{Ondulaciones denudacionales (Dmo)}

Tiene forma colinada, cóncava, inclinación suave y drenaje divergente. Se relaciona con procesos de meteorización y erosión sobre rocas blandas. Su disposición es ligeramente inclinada.

\subsubsection{Lomeríos poco bisectados (DAlpd)}

Su morfología es alomada, con cimas planas amplias y redondeadas, de laderas cortas y de forma recta, con pendientes inclinadas muy abruptas.

\subsection{Ambiente cárstico}

\subsubsection{Valle cárstico (Kvca)}

En el área de estudio se encuentra con una morfología elongada, de fondo suavemente ondulado, con bordes cóncavos bien marcados, formados por disolución de rocas calcáreas Cretácicas.

\subsubsection{Ladera cárstica (Klac)}

La ladera cárstica se caracteriza por presentarse como una superficie en declive, de morfología alomada, con pendientes inclinadas a escarpadas, de longitud corta. El patrón de drenaje es cárstico. Es común la presencia de valles ciegos.

\subsection{Ambiente fluvial}

\subsubsection{Llanura de inundación (Fpi)}

Se localiza bordeando los cauces fluviales de las quebradas locales. Su morfología plana es eventualmente inundable. Se presenta como superficie estrecha, alargada y profunda, con red de drenaje de tipo subparalelo de mediana densidad. Los procesos erosivos evidenciados son producidos por la quebrada Socomba, aportante del material cuaternario en la zona. 


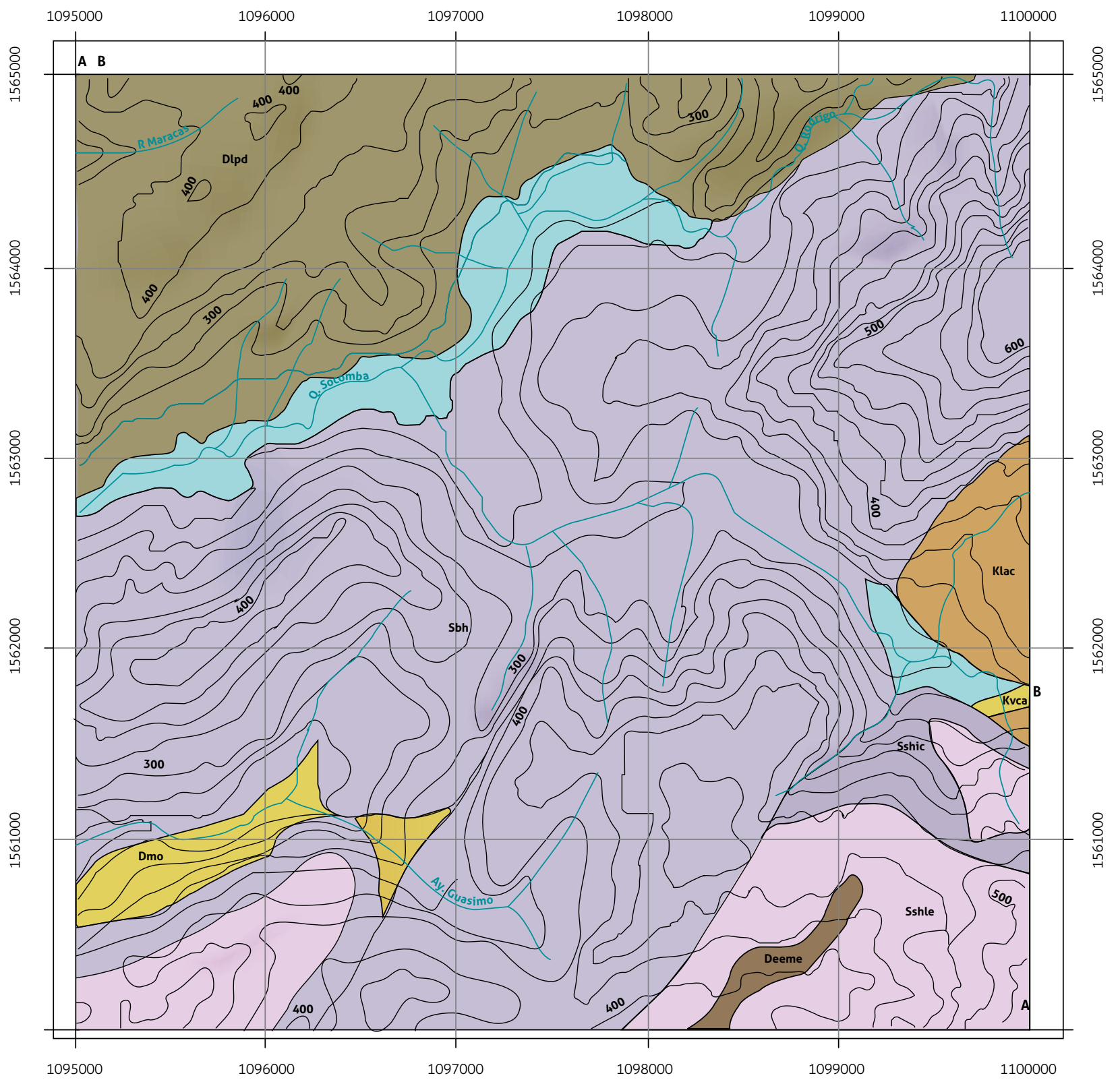

Geomorfología
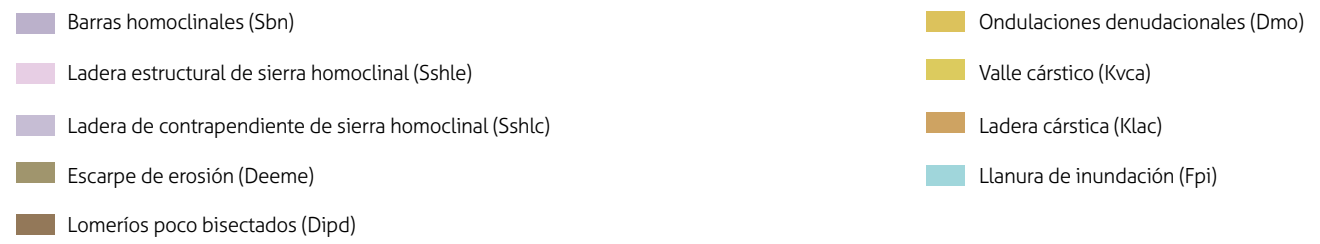

Figura 8. Geomorfología del área de estudio. Nomenclatura y colores tomados del Servicio Geológico Colombiano (2017). 


\section{Conclusiones}

Como resultado final de la cartografía, se obtuvo un mapa geológico a escala 1:25.000 que muestra la variación espacial litológica de las Formaciones Lagunitas y Aguas Blancas, del Grupo Cogollo.

La Formación Lagunitas se caracteriza por presentar una sucesión de calizas masivas fosilíferas de coloración gris oscura, con pequeñas alternaciones de lodolitas calcáreas y calizas lumaquélicas.

De la Formación Aguas Blancas se identifican con detalle el Miembro Ánimas, constituido por lodolitas calcáreas con abundante materia orgánica intercalada con calizas micríticas de color negro, que gradan horizontalmente a areniscas con cemento calcáreo de grano fino; el Miembro Tucuy, compuesto por limolitas arenosas de cemento calcáreo, areniscas de gran fino y arcillolitas calcáreas, y el Miembro Maracas, compuesto principalmente por calizas lumaquélicas masivas.

Se identificaron cuatro ambientes geomorfológicos: a) estructural, en el que se identificaron las unidades de barra homoclinal, ladera estructural de sierra homoclinal y ladera de contrapendiente de sierra homoclinal; b) denudacional, en el que se identificaron unidades como escarpe de erosión, lomeríos poco bisectados y ondulaciones denudacionales; c) cárstico, con unidades de valle cárstico y ladera cárstica, y d) fluvial, con la unidad llanura de inundación.

\section{REFERENCIAS BIBLIOGRÁFICAS}

Cáceres, C., Cediel, F. y Ujueta, G. (1976). Mapa geológico de Colombia, a escala 1:1'000.000. Bogotá: Ingeominas.

Castro, A. (1989). Petrografía básica: textura, clasificación $y$ nomenclatura de rocas. Madrid: Paraninfo.

Castro, E. (2009). Aspectos geológicos y principales consideraciones de la evaluación ambiental estratégica (diagnóstico ambiental) del distrito minero La Jagua, departamento del Cesar. Bucaramanga: UIS.

Colmenares, F. H., Mesa, A., Roncancio, J., Arciniegas, E., Pedraza, P., Cardona, A. et al. (2007). Geología de las planchas 11,12, 13, 14, 18, 19, 20, 21, 25, 26, 27, 33, 34 y 40. Proyecto: evolución geohistórica de la Sierra Nevada de Santa Marta. Bogotá: Ingeominas.

Dickinson, W. R. (1985). Interpreting provenance relations from detrital modes of sandstones. En G. G.
Zuffa (ed.), Provenance of arenites (pp. 333-361). Dordrecht: Reidel Publ. Doi: https://doi.org/10.1007/97894-017-2809-6_15.

Dunham, R. J. (1962). Classification of carbonate rocks according to depositional texture. En W. E. Ham (ed.), Classification of carbonate rocks. Memoir 1 (pp. 108121). Tulsa: AAPG.

Flügel, E. (2004). Microfacies of carbonate rocks analysis, interpretation and application. Berlin-Heidelberg: Sprmger-Verlag. Doi: https://doi.org/10.1007/978-3662-08726-8.

Folk, R. L. (1962). Clasificación de las rocas de carbonatos de acuerdo a las proporciones relativas de los tres constituyentes básicos: granos (aloquimicos), matriz micrítica y cemento, esparítico (ortoquímicos). Boletín Asociación Americana de Geólogos del Petróleo, 43.

Gardner, A. H. (1926). Suggested nomenclature and correlation of geological formations in Venezuela. Transactions of the American Institute of Mining and Metallurgical Engineers, 677-684.

Geoestudios, Ltda. (2006). Cartografía geológica cuenca Cesar-Ranchería. Informe final. Bogotá: Ministerio de Minas Y Energía, Agencia Nacional de Hidrocarburos.

Gil, A. y Martínez, H. (1990). Análisis microfacial del grupo Cogollo, y formación La Luna, cuenca Cesar-Ranchería, Guajira, Colombia (trabajo de grado, Geología). Bogotá: Universidad Nacional de Colombia, Departamento de Geociencias.

González, H., Salinas, R., Barbosa, A., Muñoz, C., Gallego, C., Quintero, D. et al.(2015). Mapa geológico de la Plancha 41 Becerril. Escala 1:100 000. Bogotá: Servicio Geológico Colombiano.

González, M., Mier, R., Arias, A., Cortés, Y., Moreno, M., Salazar, O. et al. (2008). Prospectividad de la cuenca Cesar-Ranchería. Contrato 27 de 2007 entre la Universidad Industrial de Santander y la Agencia Nacional de Hidrocarburos. Bucaramanga. Disponible en http://www.anh.gov.co/Informacion-Geologica-y-Geofisica/Estudios-Integrados-y-Modelamientos/Presentaciones\%20y\%20Poster\%20Tcnicos/ Cesar_Rancheria.pdf

Hernández, M. (2003). Memoria explicativa geología Plancha 48, Jagua de Ibirico. Escala 1:100.000. Bogotá: Ingeominas. 
Hernández Sampieri, R. F. (2014). Metodología de la investigación (6. ${ }^{\mathrm{a}}$ ed.). México: Mc Graw Hill.

Liddle, R. A. (1928). Barraquin Formation. En The geology of Venezuela and Trinidad. Fort Worth: J. P. McGowan, 108-103.

Miller, J. B. (1960). Directrices tectónicas en la sierra de Perijá y partes adyacentes de Venezuela y Colombia. Memoria. III Congreso Geológico Venezolano, Caracas.
Servicio Geológico Colombiano (2017). Mapa geomorfológico aplicado a movimientos en masa, escala 1:100.000. Sistema de Información de Movimientos en Masa (SIMMA). Bogotá: SGC.

Wilson, J. L. (1975). Carbonate facies in geologic history. Berlin: Springer. 